\title{
INVESTIGATING THE COMPATIBILITY OF POLYMERS IN COMMON SOLVENT
}

\author{
MOHSAN NAWAZ ${ }^{* 1}$ MUSA KALEEM BALOCH ${ }^{2}$,WAJID REHMAN ${ }^{1}$ \\ ${ }^{I}$ Department of Chemistry, Hazara University, Mansehra, NWFP, Pakistan. \\ ${ }^{2}$ Department of Chemistry, Gomal University, Dera Ismail Khan, NWFP, Pakistan.
}

(Received: May 16, 2009 - Accepted: November 23, 2009)

\begin{abstract}
Polymers are considered as versatile material having number of applications. However, their properties depend upon the structure, molecular mass and its distribution and the interactions among polymer-polymer and polymer-solvent molecules. Hence it is necessary to synthesize a polymer with required specification, which is a difficult, time consuming and costly process. A highly successful alternative is to blend/ mix the existing polymers for the purpose. This process needs detail knowledge about the interactions and miscibility of polymers with each other. Therefore the objective of this study is to investigate the compatibility of polymers with reference to their concentration in the blend, using static and dynamic light scattering and Rheology of the material. For the purpose Polystyrene/ Polymethylmethacrylate, Polystyrene/Polyethylene glycol and Polymethylmethacrylate / Polyethylene glycol using benzene as common solvent systems have been investigated in detail. The variation in hydrodynamic radius with concentration of polymer in the blend is found to be a good tool to ascertain the compatibility of polymers. Viscosity results also second the light scattering observations. It has been concluded that the polymer's compatibility depends upon composition of the blend and compatible with in a certain range. The compatibility play key role in the conformational behavior of the polymers blend and the techniques used is quite sensitive to identify the phase separation point.
\end{abstract}

Key words: Compatibility, Light Scattering, Blending, Viscosity, hydrodynamic radius

\section{INTRODUCTION}

Blending of polymers is widely accepted in the industry for the production of a polymeric materials with specific applications through an inexpensive route which otherwise not attainable with a single polymer. One of the important controlling parameter in this case is the degree of compatibility of the polymers blended. However, the degree of compatibility is very much dependent over the interaction between the polymeric phases of the polyblend. Therefore, varying the interactive forces among the constituents of the system one can get the material of required mechanical properties. ${ }^{1-12}$ This is the one of the reasons that miscible polymer blends are receiving more importance in industrial applications. ${ }^{13,14}$ However, the main problem associated with blending polymers is the determination of degree of compatibility. Different techniques are presently being developed to produce polymer blends and/ or determine their compatibility.

The study performed over this issue are dissolving of at least two polymers in common solvent in equal ratio and measuring the viscosity and/ or light scattering to investigate the compatibility of polymers ${ }^{15}$ Another way is to have the solution of one polymer and making the measurements by adding the other gradually. ${ }^{15-21}$ Further to it the light scattering technique has become popular for investigating the conformational behavior and dynamics of binary polymer system. ${ }^{22-25}$ Benmouna et al. ${ }^{26}$ developed a mathematical treatment to extract the required information from the dynamic light scattering spectrum of the ternary polymer solution in the semidilute regime, which has attracted a quite good number of researchers to validate the theory and interpret the results accordingly. ${ }^{27-29}$ However due to the involvement of a number of parameters, the credit of these expressions becomes doubtful and needs further exploration and simplification. On the other hand, though Polystyrene - Polymethyl methacrylate is one of the most important blend but very little work has been carried out over it and the available work in this respect is over 50:50 Polystyrene/ Polymethyl methacrylate ratio. In order to cover the whole range of polymer composition and to study the behavior of Polymethyl methacrylate blended with a polymer with and without phenyl group, we have investigated the properties of the Polymethyl methacrylate -Polystyrene-benzene and Polymethyl methacrylate -Polyethylene glycol-benzene covering the whole range of composition using viscosity and light scattering measurements.

\section{EXPERIMENTAL}

\section{Sample preparation}

The Polystyrene, Polymethyl methacrylate and Polyethylene glycol samples used in this study were purchased from E. Merck Germany. Benzene is a good solvent for these polymers. Structural and thermodynamic properties of the polymers in benzene are obtained from static light scattering measurements.
For light scattering measurements, polymer solutions were allowed to mix thoroughly assisted by gentle agitation. Dust in solutions was removed by filtering through a $0.2 \mathrm{~mm}$ Whatman Anotop 25 filter into light scattering cells, which were carefully sealed immediately after filtering.

\section{Measurement of refractive index increment}

The refractive index data of some polymer-solvent pair is obtained from polymer handbook, but in most cases we measure the data under the same experimental condition as employed in the light scattering experiment with the help of digital Hand Refractometer. The refractive index increments were obtained from the slope of the plot of refractive index versus concentration.

\section{Laser Light Scattering measurements}

The static laser light scattering measurements were carried out with DAWN EOS (Enhanced Optical System) having $30 \mathrm{~mW}$ linearly polarized GaAs (gallium arsenide) laser supplied by Wyatt Technology Corporation. The dynamic light scattering data as collected, using Wyatt QELS (Quasi Elastic Light Scattering) correlator also supplied by Wyatt Technology Corporation, at a fixed angle of $108^{\circ}$. For the purpose solutions of different compositions having various concentrations $(0-4 \%)$ were used.

\section{Viscosity Measurements}

Viscosity of the pure solvents and that of the solutions was determined, using Ostwald 's type capillary viscometers. The dimensions of capillary of the viscometers used were selected in such a way that shear effect was eliminated. The viscometers used were calibrated over the required temperature range. Measurements were made in a thermostat, manufactured by F.G Bode \& Co, laboratory equipment Hamburg, Germany, the temperature of which could be maintained to $\pm 0.01{ }^{\circ} \mathrm{C}$ of the required one. The viscosity was measured at the temperatures from 20 to $60{ }^{\circ} \mathrm{C}$. The rheological properties of all the solutions were measured at different shear rates with the help of Brookfield DV-E Viscometer at different shear rates. To keep the temperature of the experiment constant UL adopter and the thermostat supplied by Haake, Germany is used with DV-E viscometer.

\section{RESULTS AND DISCUSSION}

The blends formed by the different compositions of Polystyrene/ Polymethyl methacrylate, Polystyrene/Polyethylene glycol and Polymethyl methacrylate /Polyethylene glycol are studied by dynamic light scattering and rheometric techniques. Figure 1 represents the $\mathrm{R}_{\mathrm{H}}$ as a function of polyblend composition for various concentrations. In figure 1 it is clearly seen that the values of hydrodynamic radius first increases up to 20/80 (Polystyrene/ Polymethyl methacrylate) composition and then a regular decrease is observed. The size of the polymer first increases with the increase in composition of Polymethyl methacrylate but then decreases regularly with the increase in 
composition of Polymethyl methacrylate. The Polystyrene and Polymethyl methacrylate are incompatible, depending on the relative concentrations of Polystyrene and Polymethyl methacrylate; this could in extreme circumstances, leads to aggregation of Polystyrene in the solution. According to the literature the values of the second virial coefficient for the Polystyrene+ Polymethyl methacrylate +benzene solutions were all approximately the same. Any decrease in this parameter would be indicative of some form of aggregation of the PS leading to eventual phase separation. They also show that the second virial coefficient of Polystyrene in benzene alone is considerably different from that of the Polystyrene in the solution of Polymethyl methacrylate in benzene. This is due to additional interpolymer molecule contributions to the observed second virial coefficient of Polystyrene in these solutions. This is the reason, which is responsible for the increase in the values of hydrodynamic radius. The diffusion coefficient decreases with the increasing values of hydrodynamic radius, because it is dependent on the second virial coefficient and $R_{H}$. The most productive approach will be gained by viewing the Polystyrene chains as labeled chains in a matrix of other chains as suggested by de Gennes model. ${ }^{30,31}$ Justification for this lies in the fact that since Polymethyl methacrylate is isorefractive with benzene, then no diffusion of this species will be observed, only Polystyrene molecule appear to be moving. Since the approach of de Gennes (and of Doi and Edwards ${ }^{32}$ ) assumes the labeled chains to be chemically identical to the surrounding molecules, some differences may be expected for the Polystyrene in Polymethyl methacrylate + benzene solutions used, as the polymers are chemically different. Figures 2 show the plots of $R_{H}$ as a function of polyblend composition for Polystyrene/Poly ethylene glycol (Polystyrene/ Polythylene glycol). The same trend is observed as in the previous case for the blends of Polystyrene and Polymethyl methacrylate.
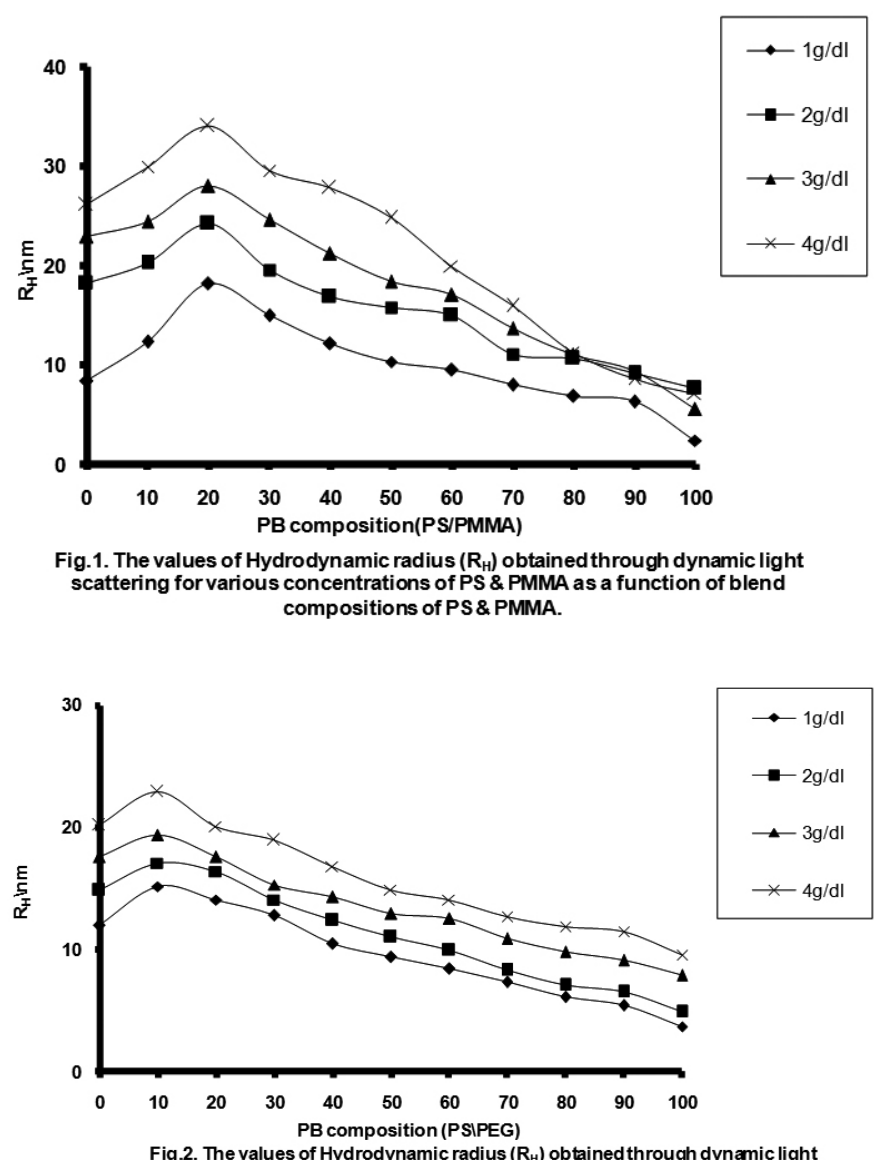
Fig.2. The values of Hydrodynamic radius $\left(R_{H}\right)$ obtained through dynamic light
scattering for various concentrations of PS \& PEG as a function of blend compositions
of PS \& PEG. of PS\& PEG. composition. From these figures it is clearly indicated that the polymer mixture changes its conformation from the flexible to the rigid rods as the concentration increases and as we move from the dilute to semidilute regime.

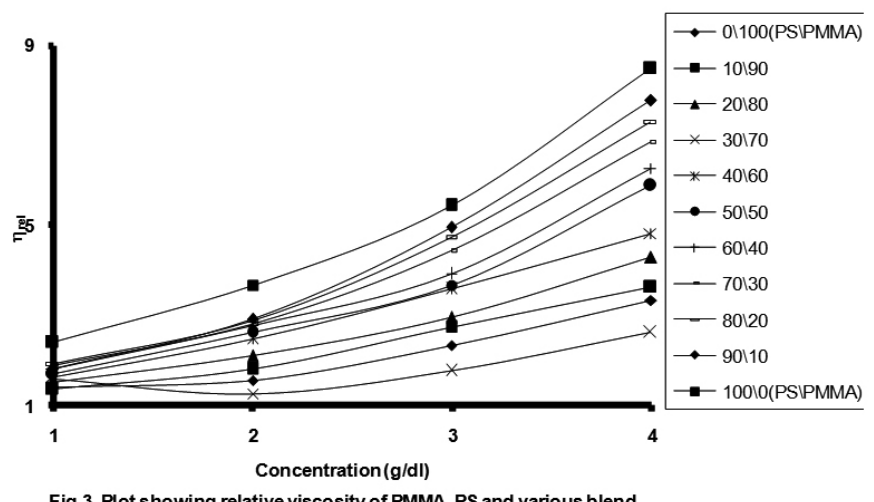

Fig.3. Plot showing relative viscosity of PMMA, PS and various blend compositions of PS/PMMA in benzene at $20^{\circ} \mathrm{C}$.

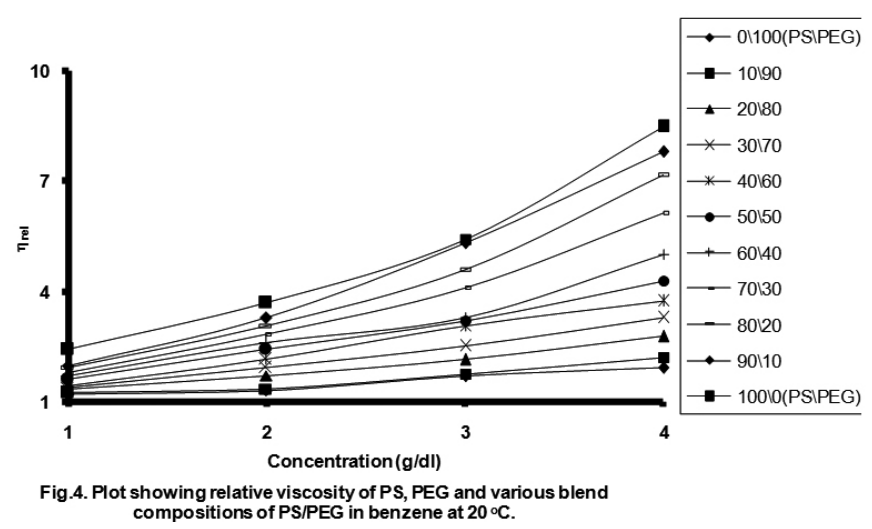

The relative viscosity data is used to calculate the specific viscosity and in turn reduced viscosity, which is then plotted as a function of concentration for every blend system to get the intrinsic viscosity. The intrinsic viscosity obtained in this way is plotted as a function of polyblend composition in figure 5 and 6 for Polystyrene/Polymethyl methacrylate and PS/PEG respectively. The figure 5 shows that intrinsic viscosity increases with the increase in the percentage of Polymethyl methacrylate. In the figure 6 the intrinsic viscosity increases with the increase in contents of Polyethylene glycol, therefore we can say that the polymer-solvent interactions are much more encouraging than those of the polymer-polymer interactions. The polymer expands with the increasing composition of Polyethylene glycol and hence an increase in intrinsic viscosity is observed. It is therefore easy that these polymers are incompatible over a wide range of composition of Polyethylene glycol. The increase in intrinsic viscosity is attributed to the fact that the phase separation in the polymer takes place and the polymer interacts with the solvent much more than it interacts with the other polymer. There are also present some interactions between the polymers which are indicated by the decrease in intrinsic viscosity of the polymer mixture. In this case it must be noted that the Polymethyl methacrylate is isorefractive with the benzene, so therefore the diffusion of Polymethyl methacrylate is not possible and all the motions are due to the molecules of the second polymer.
Figures 3 and 4 show the relative viscosity against the concentration of polymer solutions respectively for the various blend compositions of Polystyrene and Polymethyl methacrylate. The relative viscosity changes in such a way, that the curve has high values of slope almost for every 


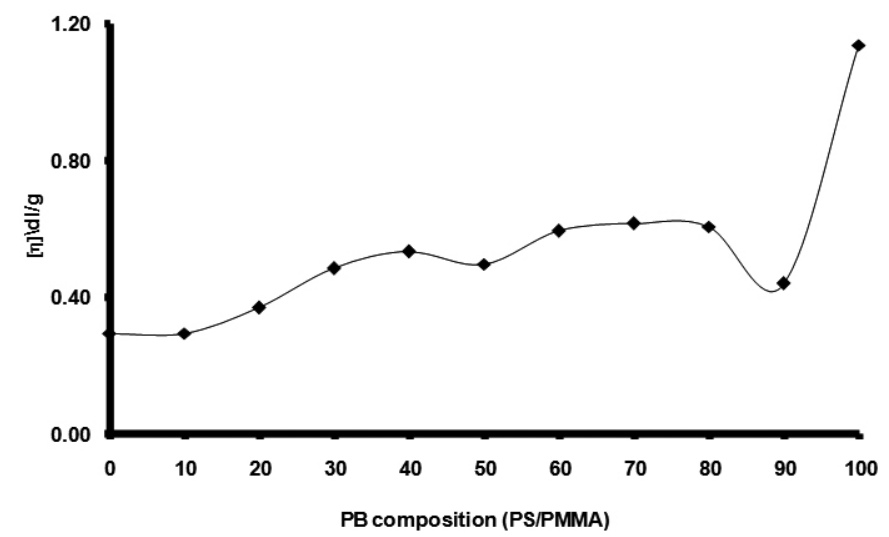

Fig. 5. Plot showing intrinsic viscosity as a function of polyblend composition of PS/PMMA at $20^{\circ} \mathrm{C}$.

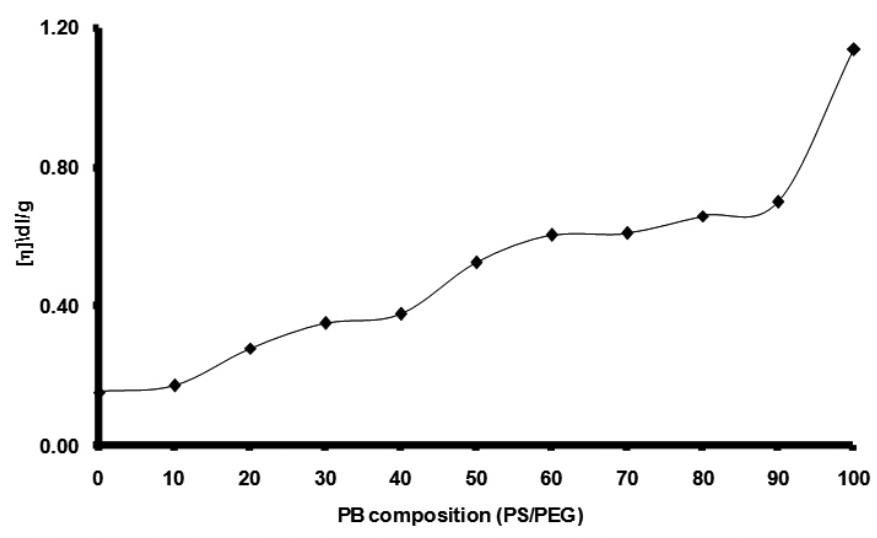

Fig. 6. Plot showing intrinsic viscosity as a function of polyblend composition of PS/PEG at $20^{\circ} \mathrm{C}$.

Various compositions of Polymethyl methacrylate/Polyethylene glycol are analyzed, using rotoviscometer in highly concentrated solutions. The range of concentration is kept between $5-10 \mathrm{~g} / \mathrm{dl}$ for each polymer. The effect of concentration on the viscosity of solution for various blend composition as determined at various speeds of the rotoviscometer and similarly the changes in viscosity with change in polyblend composition at various speeds of rotoviscometer for various concentrations of polymer solution are also studied. In almost all the cases an increase in viscosity is observed with the change in concentration and with the increase in contents of Poly ethylene glycol.

Figures $7 \& 8$ show the viscosity of Polyethylene glycol and Polymethyl methacrylate at three different speeds (RPM: Revolution per Minute) as a function of concentration of polymer solution respectively. The plots show an increase in viscosity with the increase in concentration as well as with speed of the rotor for the whole concentration studied. For Polyethylene glycol solution the lines at different speeds are widely separated from each other, while in figure 8 for Polymethyl methacrylate the lines showing viscosity lies close to each other and the lines are not widely separated from each other. But overall the viscosity seems to be independent of the speed (shear rate). The reason for this is that the change in viscosity is very little at different shear rates. Since viscosity does not depend on shear rate, the non-Newtonian behavior is explained by a decrease of entanglement viscosity. There are several theories attempting to treat the shear thinning of polymers in bulk and in solution by a purely intermolecular mechanism. A comparison of both types of theories has been provided by Graessley. ${ }^{25} \mathrm{~A}$ piece of evidence against a description based on intermolecular interactions is provided by degradation experiments. As argued by Bueche ${ }^{33}$, the forces necessary to split the C-C bonds of polymer chains are unlikely to stem from intermolecular contributions only but rather from entanglements. The predictions of Bueche's theory of degradation are at least in qualitative agreement with experiments. It has been demonstrated ${ }^{34,35}$ that the concentration affects on the frictional properties of polymer solutions can be successfully correlated with the extent of coil overlap. The influence of molecular weight upon the viscosity of polymer solutions is also related to the dimensions of macromolecular coil for dilute and concentrated solutions. This means that the statistical characteristics of an individual polymer chain determine the behavior of polymer solutions, even in the presence of a fluctuating network of entanglements. Quantitatively, this can probably be associated with the influence of the polymer chain flexibility upon the density of the entanglement network. A general concentration-viscosity relation for solutions of a given polymer is achieved ${ }^{36}$ which is independent of the molecular weight and nature of the solvent. Such master curves are different for solutions of various polymers. This is due to the fact that solutions of different polymers are not in corresponding states relative to their glass transition temperature, and the remoteness from this point depends on the concentration. The plots of viscosity versus concentration are highly linear, which indicates that solvent effects are important even in concentrated solutions and that viscosity increases more rapidly with concentration in the solvent.

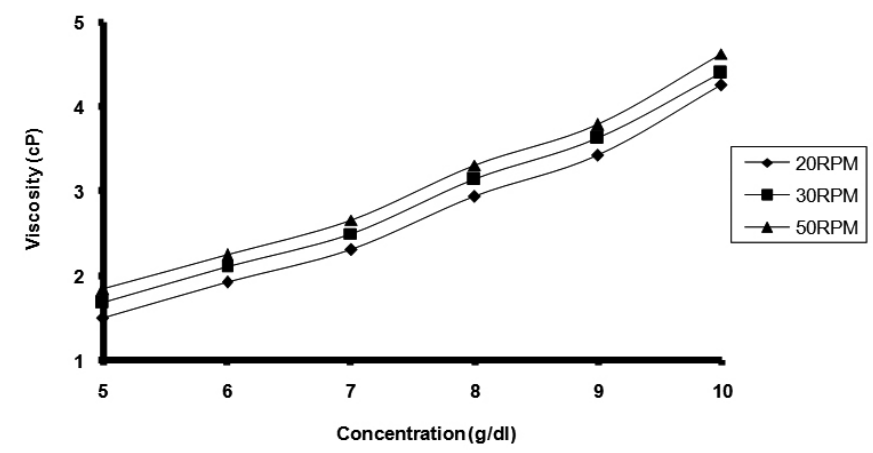

Fig. 7. Viscosity of various concentrations of PEG in benzene.

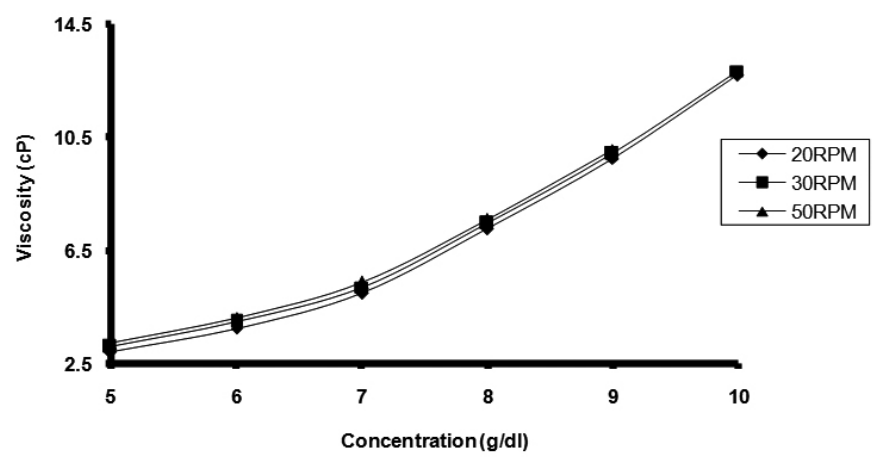

Fig. 8. Viscosity of various concentrations of PMMA in benzene.

The viscosity of various blend compositions Polymethyl methacrylate and Polyethylene glycol is plotted as a function of concentration at $25{ }^{\circ} \mathrm{C}$ at three different shear rates. All the plots are almost linear over the whole concentration range investigated and at the given shear rates studied, except the plot at the 50/50 and 40/60, which show S-type shape. It means that the polymer mixture show incompatibility at all the compositions of the mixture except, where it shows S-type plot and this is consistent with the our previous measurements, when we analyze the polymer mixtures by dynamic light scattering and capillary viscometric techniques. The minimum in these plots is observed more clearly, however, when the viscosities of the parent polymers are more nearly the same. This typical case having composition $50 / 50$ and $40 / 60$ is shown in figures 9 and 10. It should also be remembered that in flow through a capillary the shear stress varies linearly over the cross section from zero in the center to maximum value at the wall. When the shear dependence of the individual fluids brings about a viscosity reversal, the value of the viscosity ratio becomes shear dependent and changes from larger than unity to smaller than unity. This need not affect the morphology in dispersions of droplets, but it should influence the value of the viscosity of the polymer-polymer blend. The decrease in viscosity at some particular concentration of some of the blend compositions of Polymethyl methacrylate/Polyethylene glycol may also be explained by image analysis as the phase inversion concentration. 


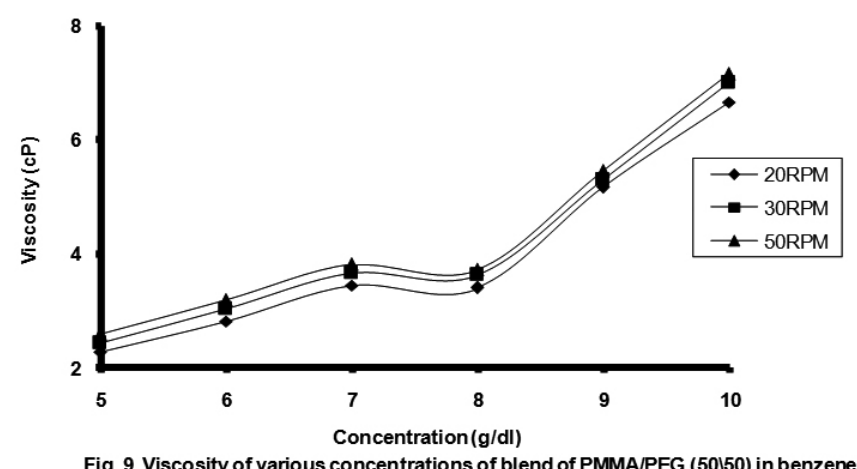

Fig. 9. Viscosity of various concentrations of blend of PMMA/PEG (50150) in benzene at different RPM.

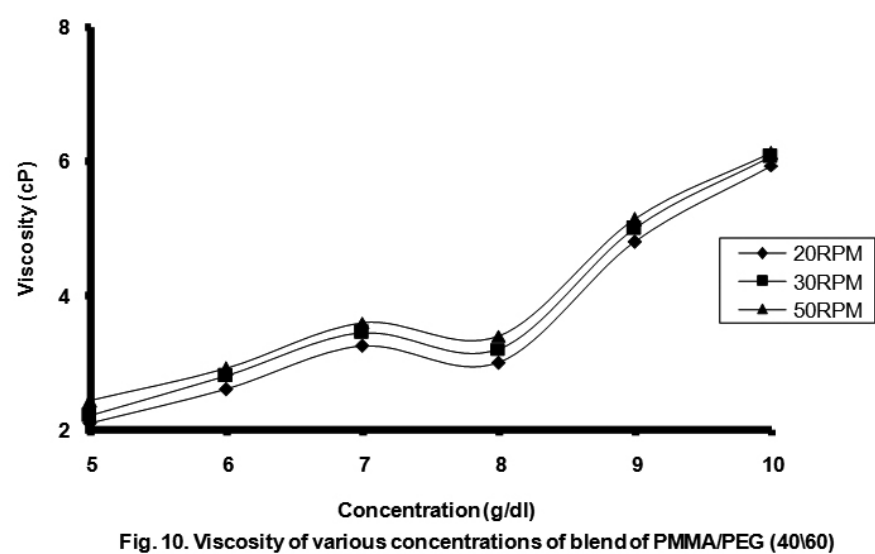
in benzene at different RPM.

The viscosity at different shear rates for different concentrations of polymer solutions is also plotted against polyblend composition of Polymethyl methacrylate/Polyethylene glycol. All the plots are linear over the whole composition range investigated, except the one shown in figure $11(8 \mathrm{~g} / \mathrm{dl}$ concentration), where it shows minimum at the particular composition of the polymer mixture. So, we can say that this is the concentration at which phase inversion takes place between the two polymers. Hence, when the viscosity of the two parent polymers differs substantially, and no viscosity minimum is observed, the viscosity of the blends are surprisingly low. As the shear stress is increased, the viscosities tend to fall below, even for dispersion of droplets. A value of the viscosity lower than that of either constituent is, however, not predicted, regardless of morphology.

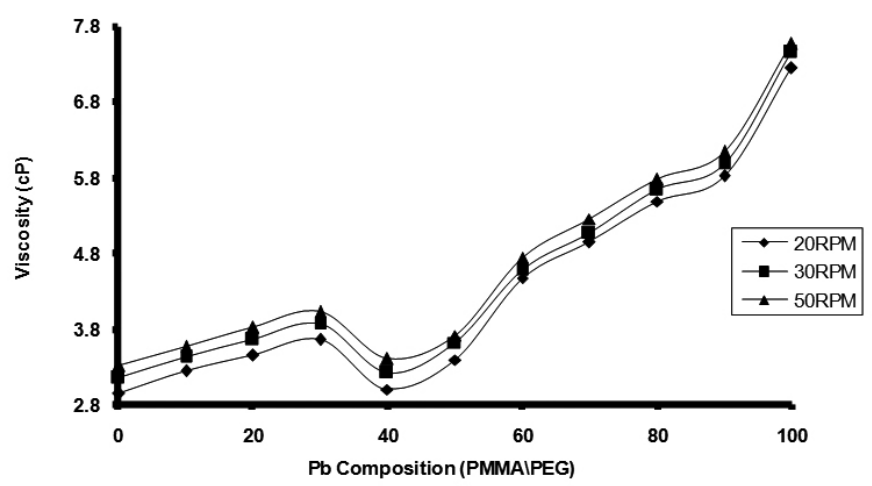

Fig. 11. Viscosity of $8 \%$ concentrated solution as a function of polyblend compositions at different RPM.

\section{CONCLUSION}

The polymer pairs used for blending in this work show partial compatibility by dissolving them in common solvent like benzene. The compatibility among these polymers is found to increase by blending them almost in equal ratios. At he two extremes when we have very little amount of one polymer and larger amount of the other, the polymer pairs are found to have very low compatibility or in other words they are incompatible to a greater extent.

\section{REFERENCES}

1. K.K.S. Hwang, C.Z. Yang, S.L. Cooper, Polym. Eng. Sci 21, 1027 (1981).

2. A. Eisenberg, P. Smith, Z.L. Zhou, Polym. Eng. Sci 22, 117(1982).

3. J.A. Miller, K.K.S. Hwang, C.Z. Yang, S.L. Cooper, J Elastomers and Plastics 15, 175 (1983).

4. P. Smith, A. J. Eisenberg, Polym. sci. Polym. Letter Edi. 21, 223 (1983).

5. Z.L. Zhou, A. Eisenberg, J. Polym. Sci. Polym. Phys. Edi. 21, 95 (1983).

6. T.A. Speckhard, K.K.S. Hwang, C.Z. Yang, W.R. Laupan, S.L. Cooper, J. Macromol. Sci. Phys B 23, 175 (1984).

7. Hwang, K.K.S.; Speckhard, T.A.; Cooper, S.L. J. Macromol. Sci. Phys B 23, 153 (1984).

8. M. Rutkowska, A. Eisenberg, Macromolecules 17, 1821 (1984).

9. M. Hara, A. Eisenberg, Polym. Eng. Sci, 24, 1306 (1984).

10. M. Hara, A. Eisenberg, Macromolecules 17, 1335 (1984).

11. S.L. Hsu, H.X. Ziao, H.H. Szmant, K.C. Frisch, J. Appl. Polym. Sci, 29, 2467 (1984).

12. K.H. Hsieh, B.J. Wong, L.W. Chen, K.C. Frisch, Proc CICHEAICHE Symposium on Modern Chemical Engineering Technoology. Taipei, Taiwan (1986) 199.

13. D.R. Paul, S. Newman, Polymer Blends, Academic Press: New York; 1, 14 (1978). L.A. Utracki, Polymer Alloys and Blends, Thermodynamics and Rheology; Carl Heanser Verlag, Munich 1990.

15. Z. Sun, C.H. Wang, J. Chem. Phys 112, 6844 (2000).

16. M.J. Meskowicz, S.L. Rosen, J. Polym. Sci. Polym. Phys. Ed. 17, 715 (1979).

17. C. Hugelin, A. Dondos, Makomol. Chem 126, 206 (1969).

18. A. Dondos, H. Benoit, Makomol. Chem 176, 3441(1975).

19. A.S. Zulkasheva, P.E. Khamzamulina, Z.K. Bakuova, E.A. Bekturov, Izv. Akad. Nauk. Kaz. SSR, Ser. Khim 26, 2 (1973)

20. P. E. Khamzamulina, Z.K. Bakuova, E.A. Bekturov, Izv. Akad. Nauk. Kaz. SSR, Ser. Khim 36, 1, (1975).

21. M. Nawaz, M.K. Baloch, Russ. J. Phy. Chem 83, 637 (2009).

22. T. Tanaka-Fukuda, H. Inagaki, Macrmolecules 12, 1229 (1979).

23. T. Tanaka, M. Nagata, H. Inagaki, Macromolecules 17, 584 (1984).

24. T. Fukuda, M. Nagata, H. Inagaki, Macromolecules 20, 654 (1987).

25. M. Nagata, T. Fukuda, H. Inagaki, Macromolecules 20, 2173 (1987).

26. M. Benmouna, H. Benoit, M. Duval, A.Z. Akcasu Macromolecules 20, 1107 (1987).

27. L. Giebel, R. Borsali, M. Rinaudo, M. Milas, Macromolecules 26, 2592 (1993).

28. R. Borsali, M. Duval, M. Benmouna, Macromolecules 22, 816 (1989).

29. L. Giebel, R. Borsali, E.W. Fisher, G. Meir, Macromolecules, 23, 4054 (1990).

30. P.G. de Gennes, Macromolecules 9, 587 (1976).

31. P.G. de Gennes, Macromolecules 9, 594(1976).

32. M. Doi, S.F. Edwards, J. C. S. Faraday II 74, 1789 (1978).

33. F. Bueche, J. Appl. Polym. Sci 4, 101 (1960).

34. L. Utracki, R. Simha, J. Phys. Chem 67, 1052 (1963).

35. J. Roots, B. Nystrom, L.O. Sundelof, B. Porsch, Polymer 20, 337 (1979).

36. V.E. Draval, J. Polym. Sci. Polym. Phys. Ed. 11, 1055, (1973). 